Dear Author,

Please, note that changes made to the HTML content will be added to the article before publication, but are not reflected in this PDF.

Note also that this file should not be used for submitting corrections. 
Research article

\title{
The appeal of sad music: A brief overview of current directions in research on motivations for listening to sad music
}

\author{
4 Q1 Annemieke J.M. van den Tol* \\ De Montfort University, School of Psychology, United Kingdom
}

\section{Article history: \\ Available online $\mathrm{xxx}$ \\ Keywords: \\ Sad music \\ Motivation \\ Appeal \\ Self-regulation \\ Adaptive \\ Mal-adaptive \\ Therapy}

A R T I C L E I N F O

Received 29 June 2015

Received in revised form 7 January 2016

Accepted 12 May 2016

\begin{abstract}
A B S T R A C T
Sadness is the most common emotion attributed to music after happiness. A recent surge in research has explored why sad music is so popular. This work aimed to summarise the results of these studies. It is indicated that sad music is listened to more commonly in sad mood and negative situation. Many different motivations have been found with regards to listening to sad music such as validating emotions, providing solace, providing rewarding emotional experiences, and aiding reflection and relaxation. With regards to listening sad music being an adaptive way to cope, some evidence in this review indicates that this may be more so the case for psychologically healthy people (rather than depressed or anxious individuals) who are also more like to apply healthy coping strategies. In contrast, findings indicate that depressed individuals and ruminators use sad music both adaptively and maladaptive. Sad music also might be particularly helpful when people are experiencing negative life events, as it enables the expression, identification, and understanding of the situation that can aid to consolation and acceptance coping. Future directions for research and the potential application of sad music listening to therapeutic settings are discussed.
\end{abstract}

(c) 2016 Elsevier Ltd. All rights reserved.

\section{Introduction}

"Emotions are set to maximize Darwinian fitness, not happiness." (Nesse, 1991; p. 37).

People generally (but not always) prefer to feel positive emotions to negative emotions, and positive emotions have a wide range of benefits, especially in terms of improving physical and mental health (Tugade, Fredrickson, \& Barrett 2004). Yet, many popular music pieces sound sad (Schellenberg, Peretz, \& Vieillard, 2008). Studies indicate that after happiness sadness is the emotion most often attributed to music (Kreutz, 2002; Lindström, Juslin, Bresin, \& Williamon, 2002). Moreover, many people listen to sad music as a means of coping with sadness and adverse negative affective states (Van den Tol \& Edwards, 2013,2014) and do so effectively (Chen, Zhou, \& Bryant, 2007; Matsumoto, 2002). This seems surprising as listening to self-perceived sad music often increases sadness (Dillman-Carpentier et al., 2008; Eerola \& Vuoskoski, 2011; Vuoskoski \& Eerola, 2012) and as sadness induced by music has been suggested to be comparable to genuine sadness, such as

\footnotetext{
* Corresponding author at: Hawthorn Building 0.30, School of Psychology, Faculty of Health \& Life Sciences, De Montfort University, Leicester LE1 9BH, United Kingdom.

E-mail address: Annemieke.vandentol@dmu.ac.uk
}

the sadness elicited by autobiographical memories (Vuoskoski \& Eerola, 2012).

But what motivates people to listen to sad music, and is this really a healthy way to cope with negative events? A recent surge in research has addressed this question. Yet so far, no manuscript has reviewed the conclusions of all these studies. Given the rise of sad sounding popular music in the last decade (Schellenberg \& von Scheve, 2012) and the availability of music given modern portable device technology, this question has important implications. The aim of this paper was to write an easily accessible paper that quickly outlines the current state of the scholarly literature into motivation for listening to sad music.

\subsection{What is sad music?}

Research indicates that people are very adept at recognizing which emotions are expressed and portrayed in music (Haack, 1980; Juslin \& Laukka, 2004; Lindström et al., 2002). And, even though small differences exist in the accuracy of perception of expressed emotions in music, the general perception of emotions in music is robust, especially for happy and sad music (Haack, 1980; Juslin, \& Laukka, 2004; Lindström et al., 2002). This broadly suggests that music that is self-identified to sound sad by an individual listeners overlaps in features with music that has been identified by research to sound sad. 
Emotion in music is broadly predicted by music features, but it is also a (subjective) product of the listener's associations with the music. In terms of the former, emotion perception in music stems partially from the features of the music, such as tempo, mode and sequence of tone use, instrument choice, dynamics, and volume (Gabrielsson \& Lindström, 2001). High sound level, fast tempo and major chords are characteristics of happy music. In contrast, low sound levels, slow pace and minor chords are characteristics of sad music (Gabrielsson \& Lindström, 2001; Juslin, \& Laukka, 2004, Khalfa, Roy, Rainville, Dalla Bella, \& Peretz, 2008). More recent results additionally emphasizes the importance of sad lyrics that are relatable for the listener in sad music (Eerola, Peltola, \& Vuoskoski, 2015; Van den Tol \& Edwards, 2013) and in music that provides solace or comfort when being sad (Ter Bogt et al., 2015, submitted for publication; Hanser, Ter Bogt, Van den Tol, Mark, \& Vingerhoets, 2016), whereas lyrics may not play such an important role in happy music.

Yet, the listener's relationship to the music (e.g., personal connection, memories and past experiences, perceived aesthetic value) and personality (e.g. empathy, absorption) undoubtedly plays an additional role in determining the depth of sadness or happiness felt when listening to the music (Van den Tol, 2012; Van den Tol \& Edwards, 2013, 2015; Van den Tol \& Ritchie, 2015). For example, in a recent experiment (Vuoskoski \& Eerola, 2012) two out of the four conditions involved listening to sad music; which was either selfidentified sad music, or unfamiliar, instrumental sad music chosen by the experimenters. It was found that trait empathy contributed to the susceptibility to sadness induced by unfamiliar music (with high empathy individuals finding it sadder), while autobiographical memories contributed to the amount of sadness induced by self-selected music. Put differently, the tone of the music may lead it to be easily identifiable as happy or sad, but the felt emotional experience is also product of the unique relationship between the listener and the piece of music.

\subsection{Motivations for listening to sad music}

A series of recent studies have tested the tendencies to like, select, and enjoy sad music. For example, several recent studies have focused on understanding the variety of different selfregulatory motivations for listening to sad music, or understanding people's attraction to sad music in general. Studies have also compared tendencies to listen to sad music, or compared sad to happy music preference across situations. In what comes next the situational characteristics that contribute to listening to sad music will first be outlined, after which the different psychological purposes that sad music may serve will be reviewed.

\subsection{Situations}

In line with what has been found about music listening in general, people report listening to self-identified sad music when travelling, when driving, reading or working, where sad music represents an optimal musical background (Taruffi \& Koelsch, 2014). Despite this overlap with happy music, a variety of different studies have indicated that people especially listen to sad music in situations in which they experience a negative emotional state. For example, the loss of a loved one has been noted to be very important as a reason for listening to self-identified sad music (Taruffi \& Koelsch, 2014; Van den Tol \& Edwards, 2013). Other situations in which people indicate to listen to self-identified sad music are situations in which people feel lonely or which they feel the need to be accepted or understood (Taruffi \& Koelsch, 2014; Van den Tol \& Edwards, 2013). When fatigued, participants also showed an increased liking for sad music (Schellenberg et al., 2008).
Many recent studies additionally provide evidence that people listen to music that portrays sadness when experiencing sadness or adverse negative affective states (Hunter, Schellenberg, \& Griffith, 2011; Matsumoto, 2002; Saarikallio \& Erkkilä, 2007; Schellenberg, Peretz, \& Vieillard, 2008; Van den Tol, 2012, Van den Tol \& Edwards, 2013, 2015; Wilhelm et al., 2013). More specific, even though people indicate that they also listen to sad music when in positive moods (Tahlier, Miron, \& Rauscher, 2013; Van den Tol \& Edwards, 2015) empirical evidence suggest that sadness rather than happiness is a stronger predictor of wanting to listen to sad music (Hunter, Schellenberg \& Griffith, 2011; Matsumoto, 2002; Van den Tol \& Ritchie, 2015). This is true when the sad mood is chronic, and when it has been experimentally induced (Chen, Zhou \& Bryant, 2007). Moreover, a series of 3 experimental studies indicate that people prefer sad music irrespective of the self-relevance of the sad mood induction - relative to happy or neutral mood induction (Taylor \& Friedman, 2015). As part of these experimental studies participants were either encouraged to imagine experiencing a loss that was relevant either to their own life or a stranger's life. The aversion for expressively happy music following a sad mood induction was partially mediated by beliefs that choosing happy music was inappropriate (e.g. not fitting the situation or the emotion) when experiencing sadness. In extension of these findings research has also found that people are less likely to choose to listen to sad music if more time has passed after a negative mood induction, people (Chen, Zhou \& Bryant, 2007) as they then become more focussed on improving their mood. In addition, resolved sadness (i.e. when a sad story has an ending) leads to increased likelihood of listening to sad, rather than to happy music, when compared with unresolved sadness (Tahlier, Miron, \& Rauscher, 2013). This is in line with the popularity of listening to sad music in situations in which negative events are difficult to change (Van den Tol, 2012, chapter 5) or situations in which people seek solace or consolation (Hanser et al., 2016; Van den Tol, Edwards, \& Heflick, 2016) such as when having to cope with the loss of a loved one.

\subsection{Sad music and self-regulation}

A recent qualitative study explored people's reasons for listening to sad music when feeling sad among a sample of sixty-five volunteers (Van den Tol \& Edwards, 2013). The authors of this study noted that in order to understand why people listen to sad music after a negative life event, we should not just look at people's expected psychological advantages for doing so, but also look at why people select a specific piece of music. Indeed, further research (Van den Tol \& Edwards, 2015) on this topic, verified that motivations for selecting a song where important for predicting expected effects of listening to sad music.

In terms of music selection strategies (music selection strategies give more information on why someone desires to select this specific piece of music) four reasons were identified. Memory Triggers refers to music selection based on perceiving that the music has high associations with past events. Connection refers to choosing to listen to music because it portrays affect or lyrics that the listener can identify with in the moment. High aesthetic value involves selecting the music based on its beauty and is linked to feeling more positive after listening (Van den Tol \& Edwards, 2015). Direction (called: Music Message in Van den Tol \& Edwards, 2013) involves choosing music that conveys a message with which the listener wants to relate.

In terms of self-regulatory goals (self-regulatory goals are what listeners hope they will achieve in terms of psychological response) several different reasons were identified: Sadness (called: Reexperiencing affect in Van den Tol \& Edwards, 2013) occurs when the music listening results in getting in touch with, intensifying or expressing feelings of sadness. Memories and social occurs when 
the music results in memory retrieval related to friends and family. Mood-enhancement involves making one feel better. Distraction refers to the use of music for distraction and keeping the mind from unwanted feelings and thoughts. Cognitions refers to an outcome in which people experience re-appraisal and thinking through problems. Lastly, Friendship is feeling like the music is a symbolic friend. One thing that should be taken into account when interpreting the above results is that this research focussed specifically on voluntarily listening to self-selected, self-identified sad music when feeling sad and hence did not represent the effects of listening to sad music in a situation where listeners are merely 'exposed' to sad sounding music.

\subsubsection{Validating feelings of sadness}

There seems to be a broad consensus among scholars that listening to sad music can be particularly helpful as a means of identifying, expressing, validating, and releasing feelings of sadness (Garrido \& Schubert, 2013; Matsumoto, 2002; Saarikallio \& Erkkilä, 2007; Schubert, 2007; Taruffi, \& Koelsch, 2014). This available effect of listening to sad music has been found to serve an important role in therapy in terms of communication of personal issues between patient and therapist, as has been found studies among grieving adolescents who received music therapy (Dalton \& Krout, 2006; McFerran, 2010a; Skewes, 2001). Findings of other research additionally suggest that musical validation of sadness (and also anger) may enable listeners to feel less alone and more understood (Hines \& McFerran, 2014) and may also serve as a means for acceptance based coping (Van den Tol, Edwards \& Heflick, 2016). Moreover, it may also be important to note that the sadness caused by music is different (e.g. less unpleasant) than sadness in everyday life, due to it not being caused as a consequence of a negative event (Scherer, 2004).

However, although some studies suggest the validation of sad emotions trough sad music to be adaptive, other research suggest it to be less adaptive. For example, Carlson et al. (2015) found that the release of anger or sadness through music that expresses these same emotions (i.e. labelled Discharge by Saarikallio \& Erkkilä, 2007) correlated mildly to anxiety and neuroticism. McFerran and Saarikallio (2014) as well as Garrido and Schubert (2015) found depressed adolescents to be more prone than healthy adolescents to couple their listening to sad (and aggressive) music with maladaptive coping tendencies such as maladaptive rumination, social isolation and inability to improve their mood. Research also shows that involuntary focus on negative thoughts (hence maladaptive) predicted music use in negative moods (Greenwood \& Long, 2009), is related to depressive symptoms (Garrido \& Schubert, 2013; Silk, Steinberg \& Morris, 2003), and that liking for sad music is increased among depressed adolescents (Hogue, Crimmins \& Kahn, 2015; although this has not been found consistently; Punkanen et al., 2011). In addition, Miranda et al. (2012) noted that shared engagement in listening to sad music with friends may both encourage increased social support and co-rumination.

In other words, examples of adaptive and maladaptive focus on emotions have been indicated in recent scholarly literature. Reflecting on the above, Saarikallio (2016, in press) noted that 'it may not necessarily be maladaptive to focus on or even intensify the current negative emotional state as long as the involvement involves reflectiveness instead of rumination, solution orientation instead of getting stuck in repetition, and focus on positive instead of negative aspects.' This theorizing resembles Trapnell and Campbell (1999) separation between maladaptive and adaptive rumination.

\subsubsection{Experiencing solace, consolation or acceptance}

Another motivation commonly mentioned in scholarly literature to explain listening to sad music is the use of sad music to experience solace, consolation, or acceptance of negative feelings and situations. In a recent survey study participants reported that they were more likely to listen to sad music than happy music when seeking consolation or in order to experience acceptance of a negative emotion or situation (Van den Tol et al., 2016), though they preferred happy music in general. Moreover, the use of music (in general) for consolation is very common. More specific, Ter Bogt et al. (submitted, 2015) conducted a survey among 1040 respondents and found that a great majority (over 60\%) of adolescents and young adults reported that they use music as a source of consolation. Results of a recent survey study (Hanser et al., 2016) additionally indicated that music is the most important source of consolation compared with other soothing behaviours.

Studies conducted with healthy populations typically suggest that listening to music that reflects one's negative mood may be a healthy act, as it provides solace or helps in gaining increased insight of a sad affective state (Hanser et al., 2016, in press; Skånland, 2013), as well as aiding in accepting these sad emotions (Van den Tol et al., 2016) and broad feelings of comfort (Van den Tol \& Edwards, 2013). More specific, Van den Tol et al. (2016) found a correlation between sad music listening with acceptance of both a negative situation and the associated negative emotions. Additionally, seeking to deal with emotions was associated with both sad music listening (for consolation) and acceptance. Moreover, Hanser et al. (2016) indicated that consoling music induces a feeling of being moved and a mixture of both positive and sad emotions.

Recent research tried to find links between psychopathology and music use for solace and found no evidence for this link (Carlson et al., 2015). This being said, Ter Bogt et al. did find higher levels of anxiety and depression and lower levels of aggression among participant that reported a higher level of listening to music for consolation. These findings may be explained as being a symptom rather, than a cause of anxiety and depression, indicating that listening to sad music for these reasons may not necessarily be maladaptive. These findings may however also indicate that music listening for consolation may be adaptive as well as maladaptive (such as in the case with listening to music to validate emotions). Future research should further explore this.

\section{Rewarding effects}

Taruffi and Koelsch (2014) have also followed up on the above line of sad music and self-regulation research. Their research was aimed at understanding the paradox of liking music that sounds sad, but did not specifically focus on sad situations. The survey that was used was based on previous research and over 700 participants volunteered in this study and one of the main focusses of the items involved the potential rewarding effects of listening to sad music. The four rewarding functions - based on the statistical relationship between items - were (from most important to least important): The reward of no "real-life" implications, meaning the pleasure listeners can take in music-evoked sadness due to its lack of contextual implications; The reward of emotion regulation, meaning the rewarding effects derived from regulation of negative moods and emotions; The reward of imagination, where musicevoked sadness has pleasurable effects due to the engagement of imaginative processes; The reward of empathy, which includes the pleasurable effects of experiencing music-evoked sadness due to mood-sharing and virtual social contact through the music. A follow up study (in the same paper) explored which of these rewards were specific to sad music. It was found that only the reward of no "reallife" implications and the reward of empathy were more applicable to sad than to happy music. On the other hand, the reward of imagination and the reward of emotion regulation did not significantly differ between sad and happy music.

Other scholars have also reported rewarding effects of listening to sad music. It has been found that listening to sad music may 
318 contribute to enhancing a negative mood, through mechanisms such as distraction and reappraisal or when the music is selected because of its intrinsic beauty (Hines \& McFerran, 2014; Van den Tol \& Edwards, 2013, 2015). With regards to these findings it may be interesting to note that Ter Bogt et al. (2010) concluded that the use of music as a tool for mood enhancement and coping is particularly present among those people who often listen to music, who find music important and those who react to it emotionally both with 7 positive and negative affect (those highly involved in music. Several rewarding effects of listening to sad (and angry music) were also reflected in responses of adult metal fans (Hines \& McFerran, 2014) who reported to have gained positive energy from metal music that helped to motivate and energize them. Listening to metal music was also mentioned to elicited feelings of courage and confidence, a sense of power, self-assurance and self-acceptance during their adolescent years. These participants also indicated that listening to metal music helped them to form an identity in their adolescence and to identify groups of others who shared similar social values and establish contact with them, despite its sad and angry nature. Qualitative responses of people's motivations for listening to sad music when feeling sad (Van den Tol \& Edwards, 2013, 2015) indicate that most of these motivations would also be relevant to sad music listening. More specific, some listeners merely indicate to listen to a particular sad song or musical group due to appreciation of the music or due to identifying with that type of music.

\subsection{No rationale or negative effect}

It has to be noted, however, that some people do listen to music that portrays negative affect without any rational motivations or expected benefits (Garrido, 2009; Garrido \& Schubert, 2011) or are mainly motivated to listen to a piece of music because they like the music or band and are expecting that listening to the music may make them feel good (Taruffi \& Koelsch, 2014; Van den Tol \& Edwards, 2013). In addition, some people also experience unexpected negative effects as a result of listening to sad music when feeling sad (Saarikallio, Gold, \& McFerran, 2015). For example a couple of participants in a qualitative study (Van den Tol \& Edwards, 2013) noted that they "expected" to feel happier after hearing a sad song that reminded them of someone, but actually felt worse. Moreover, some people generally do not enjoy listening to sad music and even avoid it due to previous negative effects of listening to it (Peltola \& Saresma, 2014).

In one recent study, Eerola, Peltola and Vuoskoski (2015) were interested in exploring people's attitudes towards sad music (rather than people's motivations for listening to it). To this end they gathered a broad sample of 358 participants Finnish participants, rather than gathering only participants that listen to sad music. The aim of this study was to explore several unaddressed aspects of the attitudes towards sad music, among a representative sample (however limitations with regards to the representative nature of this data do apply as only $26 \%$ of the invited representative sample participated). Indeed, in line with findings above they found one factor which they labelled avoidance that represented people's negative experiences with sad music. More specifically, sad music was identified to either be unpleasant, irritating, or making participants anxious or tired. Moreover, another factor emerged in the data that represented intensification of negative emotions called 'amplification'. A small correlation between this factor and the previous indicate that these items did not always represent voluntarily intensification of negative emotions. Other factors were very much in line with previous research (autobiographical, revival, appreciation, revival, and intersubjective) verifying earlier findings on motivations for listening to sad music.

Another important study worth mentioning (although not specifically about sad music) is a recent study that aimed to explore healthy and unhealthy music listening behavior (Saarikallio et al. 2015). As part of this research a correlation was found between unhealthy music listening with depression, rumination, stress and a negative correlation between unhealthy music listening with wellbeing happiness and school satisfaction of adolescence. The scale on unhealthy music listening included items such as; 'When I listen to music I get stuck in bad memories' and 'I listen to music over and over even though it makes me feel worse'. Interestingly, healthy music listening behavior also yielded small correlations with depression and rumination. Healthy music listening additionally correlated to reflection, school-satisfaction and happiness. These findings above are indicative of music use increasing when there is an increase of depression and ruminative tendencies. This probably means that the use of music among these groups of people may both be adaptive and maladaptive. Whether listening is adaptive or maladaptive may be depending on a combination between the motivation for listening and the circumstances.

\section{Conclusion and discussion}

Based on the existing body of empirical evidence, several conclusions can be drawn as to why sad music has such a high appeal. Sad music is listened to in many different situations. Looking closer at the findings on situational relevance, it can be observed that many situations in which people listen to sad music require emotional coping, such as the expressing, identification or understanding of negative emotions resulting from a negative event. Moreover, empirical evidence indicates that preference for sad music differs to preference to happy music, verifying the special role that sad music has in regulating affect.

Many different motivations and rewards have been found with regards to listening to sad music. This work indicates that, although sad music can sometimes increase sadness and is sometimes associated with negative psychological well-being, it also has a number of potentially helpful benefits such as validating emotions, providing solace, providing rewarding emotions experiences, and aiding relaxation and reflection. However, not all motivations for listening to sad music have been found to be adaptive and situational and personal factors may sometimes need to be taken into account before being able to make a conclusion as to which motivations are adaptive or maladaptive.

\section{Implications}

These findings are important as music therapists have long argued that all types of musical preferences should be accepted and utilized in therapy (McFerran 2010b). Although using a person's preferred genre and style of music is common practice in music therapy (Grocke and Wigram 2007) policy makers often scare away from allowing the use of music that has been found correlate with psychological problems (McFerran \& Baird, 2013). Many institutions restrict access to music with negative association, such as metal, angry and sad music (McFerran \& Baird, 2013). Restrictions on music selection in music therapy have serious implications for the quality of support these therapists are able to offer to their patients.

As outlined in this review, sad music especially plays an important role to people who are experiencing negative situation and have to cope with sadness (Hanser et al., 2016, in press; Taruffi \& Koelsch, 2014; Tahlier, Miron, \& Rauscher, 2013; Van den Tol \& Edwards, 2013) and for people suffering from depression or anxiety (Carlson et al., 2015; Garrido \& Schubert, 2013; Hogue, Crimmins \& Kahn, 2015; Silk, Steinberg \& Morris, 2003). Freedom to use sad music in therapy is important for music therapist when trying to build a relationship with clients and in exploration of a patients 
feelings (Dalton \& Krout, 2006; McFerran, 2010a; Skewes, 2001) as well as when finding soothing music that helps provide solace.

Finally, with regards to listening sad music being an adaptive way to cope, some evidence in this review indicates that this may be more so the case for psychologically healthy people (rather than depressed or anxious individuals) who are also more like to apply healthy coping strategies. However, as outlined above this does not mean that people who are anxious and depressed are only using maladaptive strategies to cope with sadness. The opposite is actually true when these motivations fit within an adaptive pattern for coping with a problem. Music therapists should take self-regulatory motivational and situational factors into account when interpreting patients' behavior. Moreover, therapists may also carefully monitor responses to music that people listen to. For example, listening to a sad song to work through feelings of grievance for the death of a loved one may actually be really adaptive in that situation, even when a person may generally have ruminative tendencies. To give another example, matching a sad mood with a sad song, may generally be more adaptive when it also provides solace, comfort or acceptance.

\section{Future directions}

There is ongoing debate about the use of sad music in relation to mental health. The results of this review indicate that there are no simple answers with regards to this topic. Sad music listening can have both positive and negative consequences, depending on a wide variety of factors. Based on the current review, it is suggested that the use of sad music in therapy can be beneficial when used properly. A continued exploration of the appeal of sad music will be beneficial to further unravel factors that affect short and long term effects of listening, and hence, to enable more efficient use of sad music in music therapy settings.

Recent evidence indicates that the benefits of listening to sad music depend on various situation-level and personality-level variables, as well as the motivations behind listening to sad music. However, despite the studies outlined in the current paper, little research has yet been conducted linking these factors. Moreover, scholars have yet to explore long term effects of listening to sad music, such as in terms of mental health and explore how motivation to listening to sad music is related to long-term effects. Hence, future research may focus on these topics to further insights on motivation for and effects of listening to sad music.

It also becomes clear from this review that most contemporary research on this topic has been conducted as a survey or open ended questionnaire. There hence is a pending need for research with an ecologically sound measurement. Given recent developments in music listening equipment, such studies could be conducted as an application on personal devices (such as the I-phone; Randall, Rickard, \& Vella-Brodrick, 2014).

\section{Uncited reference}

\section{Vuoskoski and Eerola (2011).}

\section{Acknowledgements}

I would like to thank. ... for proof reading this manuscript. I also would like to thank all the people with whom I have talked about this research, with whom I have collaborated on related work, and of whom I have been able to read their scholarly papers (some of which I was allowed to read pre-publication) for their contribution to this work.

\section{References}

Carlson, E., Saarikallio, S., Toiviainen, P., Bogert, B., Kliuchko, M., \& Brattico, E. (2015). Maladaptive and adaptive emotion regulation through music: a behavioral and neuroimaging study of males and females. Frontiers in Human Neuroscience, 9, 1-13. http://dx.doi.org/10.3389/fnhum.2015.00466

Chen, L., Zhou, S., \& Bryant, J. (2007). Temporal changes in mood repair through music consumption: effects of mood, mood salience, and individual differences. Media Psychology, 9, 695-713.

Dalton, T. A., \& Krout, R. E. (2006). The grief song-writing process with bereaved adolescents: an integrated grief model and music therapy protocol. Music Therapy Perspectives, 24, 94-107.

Dillman-Carpentier, F. R., Brown, J. D., Bertocci, M., Silk, J. S., Forbes, E. E., \& Dahl, R. C. (2008). Sad kids, sad media? Applying mood management theory to depressed adolescents' use of media. Media Psychology, 11, 143-166.

Eerola, T., \& Vuoskoski, J. K. (2011). A comparison of the discrete and dimensional models of emotion in music. Psychology of Music, 39, 18-49.

Eerola, T., Peltola, H.-R., \& Vuoskoski, J. K. (2015). Attitudes towards sad music are related to both preferential and contextual strategies. Psychomusicology: Music, Mind, and Brain, 25, 116-141.

Gabrielsson, A., \& Lindström, E. (2001). The influence of musical structure on emotional expression. In P. N. Juslin, \& J. A. Sloboda (Eds.), Music and emotion: theory and research (pp. 223-248). New York: Oxford University Press.

Garrido, S., \& Schubert, E. (2011). Individual differences in the enjoyment of negative emotion in music: a literature review and experiment. Music Perception, 28, 279-295.

Garrido, S., \& Schubert, E. (2013). Adaptive and maladaptive attraction to negative emotions in music. Musicae Scientiae, 17, 147-166.

Garrido, S., \& Schubert, E. (2015). Music and people with tendencies to depression. Music Perception: An Interdisciplinary Journal, 32, 313-321.

Garrido, S. (2009). Rumination and sad music: a review of the literature and a future direction. Proceedings of the 2nd international conference on music communication science, 20-23.

Greenwood, D. N., \& Long, C. R. (2009). Mood specific media use and emotion regulation: patterns and individual differences. Personality and Individual Differences, 46, 616-621.

Grocke, D., \& Wigram, T. (2007). Receptive methods in music therapy. London: Jessica Kingsley.

Haack, P. A. (1980). The behavior of music listeners. In D. Hodges (Ed.), Handbook of music psychology (pp. 141-182). Lawrence, KS: National Association for Music Therapy.

Hanser, W. E., Ter Bogt, T. F. M., Van den Tol, A. J. M., Mark, R. E., \& Vingerhoets, A. J J. M. (2016). Consolation through music: a survey study. Musicae Scientiae, in press.

Hines, M., \& McFerran, K. S. (2014). Metal made me who I am: seven adult men reflect on their engagement with metal music during adolescence. International Journal of Community Music, 7, 205-222.

Hunter, P. G., Schellenberg, E. G., \& Griffith, A. T. (2011). Misery loves company: mood-congruent emotional responding to music. Emotion, 11, 1068-1072.

Juslin, P. N., \& Laukka, P. (2004). Expression, perception: and induction of musica emotions: a review and a questionnaire study of everyday music listening. Journal of New Music Research, 33, 217-238.

Khalfa, S., Roy, M., Rainville, P., Dalla Bella, S., \& Peretz, I. (2008). Role of tempo entrainment in psychophysiological differentiation of happy and sad music. International Journal of Psychophysiology, 68, 17-26.

Kreutz, G. (2002). Jede Sehnsucht hat eine Melodie: Basisemotionen in der Musik und im Alltag. ['Every longing carries a melody': basic emotions in music and everyday life]. Musikpsychologie, 16, 66-83.

Lindström, E., Juslin, P. N., Bresin, R., \& Williamon, A. (2002). Expressivity comes from within your soul: a questionnaire study of music students' perspectives on expressivity. Research Studies in Music Education, 20, 23-47.

Matsumoto, J. (2002). Why people listen to sad music: effects of music on sad moods. Japanese Journal of Educational Psychology, 50, 23-32.

McFerran, K. S., \& Baird, K. (2013). Is music really my best friend? Reflections of two maturing women on one's relationship with music. In L. Ole Bonde, E. Ruud, M. S. Skånland, \& G. Trondalen (Eds.), Anthology (6) musical life stories: narratives on health musicking (pp. 117-138). Oslo: Centre for Music and Health, Norwegian Academy of Music.

McFerran, K. S. (2010a). Tipping the scales: a substantive theory on the value of group music therapy for supporting grieving teenagers. Qualitative Inquiries in Music Therapy, 5, 1-42.

McFerran, K. S. (2010b). Adolescents, music and music therapy: methods and techniques for clinicians, educators and students. London: Jessica Kingsley Publishers.

Nesse, R. M. (1991). What good is feeling bad? The Sciences, 31, 30-37.

Peltola, H.-R., \& Saresma, T. (2014). Spatial and bodily metaphors in narrating the experience of listening to sad music. Musicae Scientiae, 18, 292-306. http://dx. doi.org/10.1177/1029864914536199

Randall, W. M., Rickard, N. S., \& Vella-Brodrick, D. A. (2014). Emotional outcomes of regulation strategies used during personal music listening: a mobile experience sampling study. Musicae Scientiae, 18, 275-291.

Saarikallio, S., \& Erkkilä, J. (2007). The role of music in adolescents' mood regulation. Psychology of Music, 35, 88-109.

Saarikallio, S., Gold, C., \& McFerran, K. (2015). Development and validation of the healthy-unhealthy music scale. Child and Adolescent Mental Health, 20, 210-217.

(25)

525
526
527
528

$$
\text { , }
$$

\section{.}

3

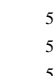

\section{.}$$
\text { . }
$$

.

\section{.}

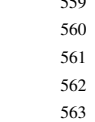

$$
\text { w }
$$

\section{6}

$$
\text { 晜 }
$$

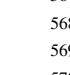 \\ .}

\section{.}

\section{7}

$$
57
$$$$
575
$$

\section{7}


